\title{
MRI-Based 3-Dimensional Visualization Workflow for the Preoperative Planning of Nephron-Sparing Surgery in Wilms' Tumor Surgery: A Pilot Study
}

\author{
Matthijs Fitski $\mathbb{D}^{\mathrm{D}}{ }^{1}$ Jene W. Meulstee $\mathbb{C}^{\mathrm{D}},{ }^{2}$ Annemieke S. Littooij $\mathbb{D}^{\mathrm{D}},{ }^{3,4}$ Cornelis P. van de Ven, ${ }^{1}$ \\ Alida F. W. van der Steeg, ${ }^{1}$ and Marc H. W. A. Wijnen ${ }^{1}{ }^{1}$ \\ ${ }^{1}$ Department of Pediatric Surgery, Princess Máxima Center for Pediatric Oncology, 3584 CS Utrecht, Netherlands \\ ${ }^{2} 3 D$ Lab, Radboud University Medical Center, 6525 GA Nijmegen, Netherlands \\ ${ }^{3}$ Department of Radiology and Nuclear Medicine, University Medical Center Utrecht/Wilhelmina Children's Hospital, \\ 3584 CX Utrecht, Netherlands \\ ${ }^{4}$ Department of Radiology and Nuclear Medicine, Princess Máxima Center for Pediatric Oncology, 3584 CS Utrecht, Netherlands
}

Correspondence should be addressed to Marc H. W. A. Wijnen; m.h.w.wijnen-5@prinsesmaximacentrum.nl

Received 10 July 2020; Revised 21 October 2020; Accepted 29 October 2020; Published 9 November 2020

Academic Editor: Zhihan Lv

Copyright @ 2020 Matthijs Fitski et al. This is an open access article distributed under the Creative Commons Attribution License, which permits unrestricted use, distribution, and reproduction in any medium, provided the original work is properly cited.

Purpose. Due to the size and localization of Wilms' tumor (WT), nephron-sparing surgery (NSS) is only possible in a limited number of cases. When NSS is considered, the surgeon preoperatively requires a thorough understanding of the patient-specific anatomy to prevent positive surgical margins and other complications. Through a collaboration between the radiology and pediatric surgery departments and 3D imaging specialists, a 3D visualization workflow was developed to improve preoperative planning of NSS for WT patients. Methods. The 3D visualization workflow combines a MRA sequence, a segmentation protocol, and augmented reality (AR) visualization, additional to in-house 3D printing. A noncontrast-enhanced MRA scan was added to the MRI protocol. MRI sequences were segmented with a segmentation protocol in an open-source software package. The resulting 3D models were visualized in AR with a HoloLens and 3D print. Results. In a pilot study, five WT patients eligible for NSS were preoperatively planned through the $3 \mathrm{D}$ visualization workflow. AR visualization software was fast and free to use and allowed adequate handling of the 3D holograms. The 3D printed models were considered convenient and practical for intraoperative orientation. The patient-friendly, fast, and low-cost 3D visualization workflow was easily implemented and appeared to be valuable for the preparation of NSS. Conclusion. This pilot study demonstrates how a strong collaboration between the pediatric surgery and radiology departments and 3D imaging specialists will help to shape the future of pediatric oncological surgery. This 3D visualization workflow aims to prepare pediatric oncological surgeons for nephron-sparing surgery in patients with Wilms' tumors.

\section{Introduction}

Wilms' tumor (WT) is the second most common abdominal pediatric tumor in Europe, with children being diagnosed at a median age of approximately 3.5 years [1]. In accordance with the International Society for Pediatric Oncology-Renal Tumor Study Group (SIOP-RTSG) Umbrella treatment protocol, therapy generally consists of neoadjuvant chemotherapy, followed by radical nephrectomy and adjuvant chemotherapy [2]. In contrast to a radical nephrectomy for local treatment, nephron-sparing surgery (NSS) can be used for nephrogenic preservation. This helps to protect the patient from excessive functional parenchymal loss in the future [3]. NSS is a technically demanding procedure and it requires a thorough preoperative understanding of the patient-specific renal anatomy and intraparenchymal vasculature [4]. Patients with bilateral disease, with or without a predisposing syndrome, might be eligible for NSS depending on the tumor location, size, and infiltration. Due to the risk of a positive surgical margin with 
NSS, unilateral nonsyndromic patients are treated with a radical nephrectomy. However, in order to prevent late effects of a radical nephrectomy at a young age, these patients might be still considered for NSS if they have a small lesion at a favorable location at the moment of diagnosis [2]. Therefore, careful selection and preoperative planning are crucial to ensure a positive oncological outcome in combination with low morbidity.

For the preoperative planning of NSS, patient-specific 3-dimensional (3D) anatomical models are increasingly used [5-7]. This is due to the improved and more accessible imaging, segmentation, and visualization techniques. $3 \mathrm{D}$ printing is a visualization technique which can be used to visualize these patient-specific models. The positive effects of $3 \mathrm{D}$ printed anatomical models in adults have been described and include reducing blood loss, reducing intraoperative complications, and improving patient education [5]. In renal surgery for pediatric oncology, 3D printed anatomical models are not frequently used and if so, only on a case-by-case basis [3, 8-11]. Regarding NSS, 3D printed models are mainly useful for assisting in planning the vascular dissection. However, the vasculature information in current $3 \mathrm{D}$ models remains poor, primarily due to low image quality [8]. Retrospectively, personalized $3 \mathrm{D}$ anatomical models have shown a significant improvement of the anatomical understanding for the renal artery, vein, tumor, and urinary collecting system and may potentially help pediatric surgeons prepare for NSS [12]. The models were limited due to low image quality and modelling techniques which are labor-intensive, require a long processing time, and are expensive.

High-quality imaging is crucial for high-fidelity anatomical models, as the imaging quality primarily determines the model quality. Preoperative magnetic resonance imaging (MRI) is already a vital part of the SIOP-RTSG Umbrella treatment protocol and can be used for 3D visualization, as Wake et al. have previously shown for renal cancer in adults [13]. An additional computed tomography angiography (CTA) scan can be performed for high quality arterial imaging, yet it is undesirable due to the radiation and contrast administration. Moreover, an additional CTA scan prolongs the preoperative workup which is already considered highly stressful for pediatric patients. Therefore, techniques solely based on the preoperative MRI are favorable. Wake et al. reported challenges in standardized high-resolution imaging and were also limited by the manual segmentation procedure. Image processing took around 7 hours and 3D printing costs were around $\$ 1000$ (US dollar) per model. To overcome these limitations, our aim is to develop a 3D visualization workflow in which imaging, segmentation, and visualization techniques are combined, to suit the specific preoperative requirements needed for planning NSS of WT in pediatric patients.

\section{Materials and Methods}

In close collaboration with the departments of radiology and pediatric surgery, 3D imaging specialists have designed a new 3D visualization workflow for the preoperative planning of NSS in WT patients. In this workflow, we addressed the limitations in usability and model quality previously described in literature. An overview of the proposed workflow is given in Figure 1. The following sections describe the employment of the noncontrast-enhanced MRA (NC-MRA) sequence, segmentation protocol, and visualization with Augmented Reality (AR) and with 3D printing.

2.1. Imaging. The standard pediatric kidney tumor MRI protocol was performed at presentation and after the neoadjuvant chemotherapy in accordance with the SIOP-RTSG Umbrella protocol. A 1.5 Tesla system (Achieva, Philips Medical Systems, Best, Netherlands) was used for all patients. The imaging protocol consisted of coronal 3D T2weighted $(-\mathrm{W})$ sequence, fat-suppressed $\mathrm{T} 1-\mathrm{W}$ sequence, diffusion-weighted imaging ( $b$ values of at least 0,100 , and $800 \mathrm{~s} / \mathrm{mm}^{2}$ ), and a fat-suppressed T2-W sequence. Before administering the contrast agent, the NC-MRA sequence was acquired. During the administration of intravenous contrast (Gadovist, Bayer Pharma, Berlin, Germany, at a dose of $0.1 \mathrm{mmol} / \mathrm{kg}$ body weight), a $4 \mathrm{D}$ contrast-enhanced MRA was acquired, after which a postcontrast fat-suppressed T1-W sequence was performed. Children were awake, sedated, or under anesthesia depending on their ability to cooperate. Hyoscine butylbromide (Buscopan, Boehringer Ingelheim Limited, Bracknell, UK) was administered at an intravenous dose of $0.4 \mathrm{mg} / \mathrm{kg}$ body weight to reduce the peristaltic artefacts.

The used NC-MRA is an inflow-enhanced balanced Steady State Free Precession (b-SSFP) sequence. This sequence has a unique T2/T1 contrast, which has a high contrast for blood. The sequence is scanned with a high reconstructed resolution $(0.56,0.56,1 \mathrm{~mm})$, takes 3-5 minutes to scan, is independent of direction, and does not require a contrast agent. An inversion time (TI) of $450 \mathrm{~ms}$ was used. If $450 \mathrm{~ms}$ was not possible due to a fast heart rate, the TI was lowered based on the maximal allowed TI ( $90 \%$ of the R-R interval). The cardiac trigger was set on a heartbeat measured preferably with a three-lead electrocardiogram or else with a physiological pulse unit. The transverse field of view (FOV) was set parallel to the renal artery of the affected kidney in the coronal view using the $3 \mathrm{D}$ T2-W sequence. The FOV encompasses the complete intraparenchymal arterial branch. A saturation band was positioned below the lower pole of the kidneys and a fat saturation band was positioned at the ventral aspect of the abdomen. This allowed saturation of signal from the vena cava and abdominal fat.

2.2. Segmentation. A selection of the MRI sequences (3D T2-W, NC-MRA, postcontrast fat-suppressed T1-W) was used to perform the segmentation in open-source software package 3D Slicer 4.10.2. A standardized protocol was developed for the segmentation of the arteries, veins, urine collecting system (UCS), tumor, and kidney. Firstly, the NCMRA sequence was used to compute the segmentation of the arteries through an intensity-based threshold technique. The "scissor" tool was used to remove artefacts and the resulting 

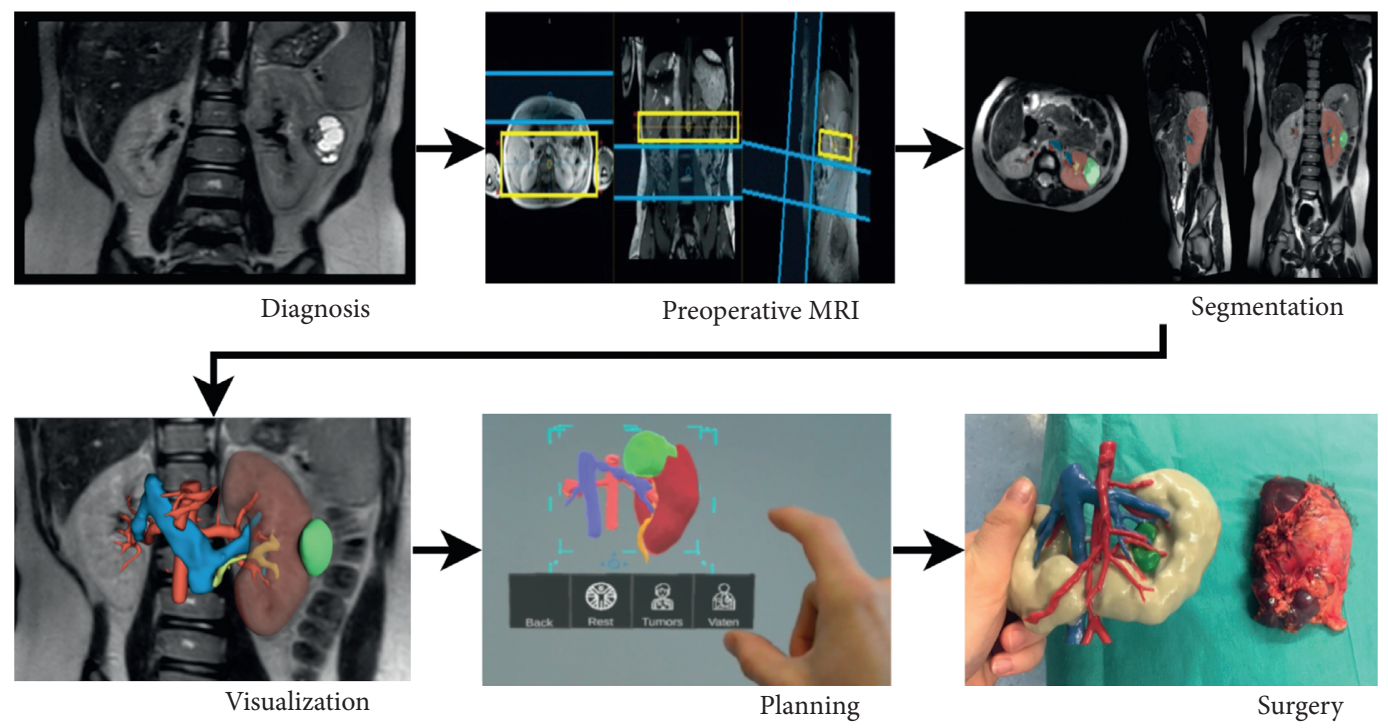

FIGURE 1: Schematic overview of the proposed preoperative 3D visualization workflow. After diagnosis and neoadjuvant chemotherapy, a WT patient receives a preoperative MRI. A high-resolution noncontrast-enhanced MRA sequence is added to the protocol to allow for visualization of the intraparenchymal arteries. The MRI scans are segmented through a standardized segmentation protocol in 3D Slicer resulting in patient-specific $3 \mathrm{D}$ anatomical models. The $3 \mathrm{D}$ models are displayed for preoperative planning in augmented reality through a HoloLens. 3D printed models can be brought inside the operating theater.

model was smoothed with a Gaussian filter. The arterial segmentation was used as an overlay in the T2-W sequence in order to differentiate intraparenchymal arteries and veins. Subsequently, the veins and UCS were segmented with an intensity-based brush in the aforementioned sequence. The postcontrast fat-suppressed T1-W sequence was used for the segmentation of the tumor and kidney with a Grow-Cut algorithm [14]. This algorithm uses labels in the area of the tumor, kidney, and background in several slices in order to compute the border between the three labels. The results were filtered with a joint-smoothing filter. Any under- or oversegmentations were manually corrected. The resulting segmentations were exported as a stereolithography (.STL) file.

2.3. Visualization. In order to visualize the $3 \mathrm{D}$ models in the STL format, the T2-W images (DICOM format) were added to AR software developed in Unity 5.5.2 (Unity Technologies, San Francisco, CA, USA). AR software ensured that the 3D models and MRI images were spatially aligned. Subsequently, AR software computed a patient-specific container which could be uploaded to a visualization library installed on the head-mounted display.

Preoperatively, the surgeon reviewed the patient-specific AR hologram in a real-world setting to prepare for surgery. The surgeon had full control over the hologram: the surgeon could translate, rotate, and scale the hologram. Additionally, the transparency of the individual anatomical models could be adjusted, and the individual models could be removed. It was possible to look at the T2-W MRI in three different planes (transverse, sagittal, and coronal) in order to correlate the orientation of the $3 \mathrm{D}$ models with the more commonly known MRI images. The AR-display from the HoloLens could be shared on a PC through a live stream to allow for an interactive discussion by the user and observers. This visualization technique has been free of costs after development of software and purchasing the HoloLens.

To create physical anatomical models, an Ultimaker S5 dual-extrusion printer was used with a Fused Filament Fabrication technique. To allow visualization of the renal pelvis, the $3 \mathrm{D}$ modelled kidney could be bisected in Meshmixer 3.5.474 (Autodesk, Inc., San Francisco, CA, USA) with a plane cut prior to printing. The resulting models were printed with a fine layer profile of $0.1 \mathrm{~mm}$ thick, infill density of $30 \%$ (zigzag infill pattern), and support overhang angle of $60^{\circ}$. The models were printed with two different colors which allowed us to improve the contrast of specific anatomical regions. 3D printed models allowed the surgeons to get a sense of the tumor size, and the model could be taken into the OR by an assistant to help the surgeon navigate during the procedure.

\section{Results}

Between May 2019 and August 2019, a pilot study was performed. Five patients were considered for NSS, and their surgeries were preoperatively planned with the $3 \mathrm{D}$ visualization workflow in addition to the standard protocol. The patients' ages ranged from 2 to 8 years (mean $5.2 \pm 1.4$ years). Patient demographics, tumor characteristics, and relevant technical outcomes for each patient are described in Table 1.

The mean additional scanning time required for the NCMRA sequence was 04:12 \pm 00:36 minutes. The mean segmentation time was $41 \pm 19$ minutes, which seemed closely correlated to the number and size of the lesions. The mean volume of the resulting tumor 3D model was $12.2 \pm 20.5 \mathrm{ml}$ per lesion. Average 3D printing time was roughly 20 hours. 
TABLE 1: Preoperative patient demographics, tumor characteristics, and pathologic outcomes for each patient together with the noncontrastenhanced MRA (NC-MRA) scanning duration, the duration of the complete segmentation, the tumor volume derived through 3D Slicer, and $3 \mathrm{D}$ printing time and costs.

\begin{tabular}{|c|c|c|c|c|c|}
\hline & Patient 1 & Patient 2 & Patient 3 & Patient 4 & Patient 5 \\
\hline Gender (M/F) & $\mathrm{F}$ & $\mathrm{F}$ & $\mathrm{M}$ & $\mathrm{F}$ & $\mathrm{F}$ \\
\hline Age (Y) & 8 & 2 & 4 & 5 & 3 \\
\hline Disease & $\begin{array}{l}\text { Unifocal } \\
\text { right }\end{array}$ & $\begin{array}{l}\text { Unifocal right, bilateral } \\
\text { nephroblastomatosis }\end{array}$ & Unifocal left & $\begin{array}{l}\text { Bilateral, } \\
\text { multifocal }\end{array}$ & $\begin{array}{c}\text { Bilateral, } \\
\text { nephroblastomatosis }\end{array}$ \\
\hline Syndrome & - & Beckwith-Wiedemann syndrome & $\begin{array}{l}16 \mathrm{p} 12.2 \\
\text { deletion }\end{array}$ & - & WT-1 mutation \\
\hline Procedure & NSS & NSS & NSS & NSS & NSS \\
\hline $\begin{array}{l}\text { NC-MRA scanning } \\
\text { duration (min: sec) }\end{array}$ & $04: 50$ & $03: 55$ & 03:55 & $04: 50$ & $03: 28$ \\
\hline Segmentation time (min) & 29 & 41 & 34 & 73 & 28 \\
\hline $\begin{array}{l}\text { Volume tumor } \\
\text { segmentation }(\mathrm{ml})\end{array}$ & 1.4 & 4.0 & 69.2 & $\begin{array}{c}24 ; 17 ; 1.2 ; 0.3 ; 3.4 ; \\
11.6 ; 0.12\end{array}$ & 2.3 \\
\hline $\begin{array}{l}\text { 3D printing time } \\
\text { (hours:min) }\end{array}$ & $18: 39$ & $15: 41$ & $16: 39$ & $31: 26$ & $18: 10$ \\
\hline $3 \mathrm{D}$ printing cost $(€)$ & 3.58 & 2.57 & 3.06 & 5.80 & 3.27 \\
\hline
\end{tabular}

NSS = nephron-sparing surgery.

Manual removal of supporting structures during the postprocessing took about half an hour.

The MRA sequence added to the preoperative MRI was successful in all patients. The segmentation and visualization in AR were performed within a day after the preoperative MRI. Figure 2 shows a 2D rendering in 3D Slicer, a 3D hologram visualized with the head-mounted display, and a $3 \mathrm{D}$ print of a 3D anatomical model, all of the same patient. The $3 \mathrm{D}$ printers generally printed the models overnight allowing ample time for the surgical team to assess the $3 \mathrm{D}$ models. The arterial models visualized the intraparenchymal arteries up to the second or third segmental arterial branch. The level of detail of the vein and UCS 3D models was noticeably less than the level of detail of the arterial models. However, surgeons considered the vein and UCS 3D models supportive and additional to the MRI imaging.

The AR visualization allowed the surgeons to assess the depth of resection with regard to renal arteries, veins, and UCS. Being able to scale, move, rotate, and walk around the hologram was very useful for the understanding of the patient-specific anatomy. Intraoperatively, an assistant showed the $3 \mathrm{D}$ printed models to visualize the location and rotation of the tumor in relation to the renal parenchyma. This proved to be mainly useful in the patients with multiple lesions.

\section{Discussion}

A combined imaging, protocolled segmentation, and visualization workflow resulted in patient-specific 3D anatomical models for the preoperative planning of WT patients. The 3D visualization workflow aimed to help pediatric surgeons improve their understanding of anatomical relationships and orientation. Because of the preparation time of about 1 hour and a printing time of 20 hours, this $3 \mathrm{D}$ visualization workflow can be completed within a limited timeframe of two days between the preoperative MRI and surgery. Additionally, it is inexpensive as the head-mounted display costs 3000 euros, and all software packages are opensource or self-developed. Limitations in previous studies on 3D modelling for WT included low image quality, long segmentation, and long visualization processing. Wake et al. reported a $3 \mathrm{D}$ printing cost per model of $\pm \$ 1000$ (US dollars), a segmentation time of \pm 7 hours, and 3D printing time of \pm 10 hours [13]. Wellens et al. reported an average cost of \$400 USD per printed model and a segmentation and $3 \mathrm{D}$ printing manufacturing time of 4 to 5 days [12]. In this pilot study, we have addressed these limitations through the development of our own innovative workflow in collaboration with the departments of pediatric surgery, radiology, and 3D imaging specialists. The segmentation protocol of the workflow allowed for fast 3D modelling and the HoloLens proved to be a fast and useful visualization tool. Our $3 \mathrm{D}$ printing technique was slow ( \pm 20 hours) because of the fine layer profile $(0.1 \mathrm{~mm}$ thickness $)$. Increasing the layer profile would decrease the printing time significantly. Our in-house 3D printer is limited in color and materials, yet it was of sufficient quality, and with an average price of \pm 3.50 euros and machine cost of 5500 euros, it was considered a sustainable technique.

It is difficult to quantify the advantages of $3 \mathrm{D}$ anatomical models for the preoperative planning of NSS for WT. An increase in surgical confidence for NSS for WT has been shown retrospectively [12], but quantifying the advantage remains subjective [12]. In adults, $3 \mathrm{D}$ printed renal models based on preoperative MRI scans could help during surgical decision-making [6]. Moreover, 3D printed models did allow adult surgeons to improve their translation from $2 \mathrm{D}$ CT and MRI data into 3D anatomical relationships, which appeared more relevant in smaller lesions [15]. Although the results from these studies may not be directly applicable to children because image quality is generally superior in adults [16], we expect that $3 \mathrm{D}$ printed models could help in the preparation of pediatric oncologic surgery. The clinical advantage of the described $3 \mathrm{D}$ visualization workflow for children may be quantified in the future. We need to 


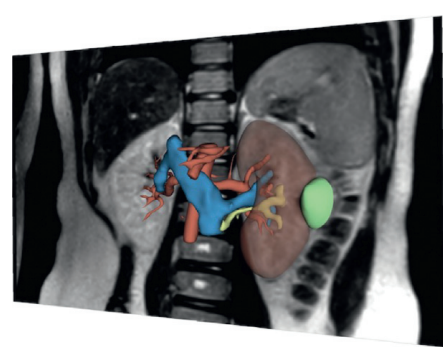

(a)

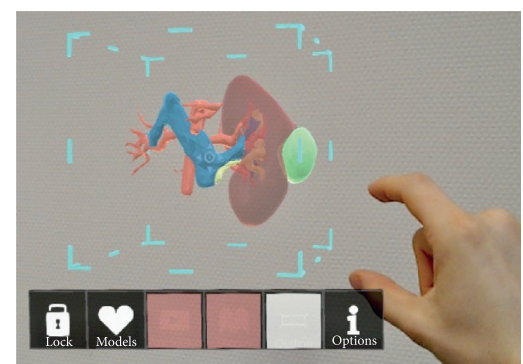

(b)

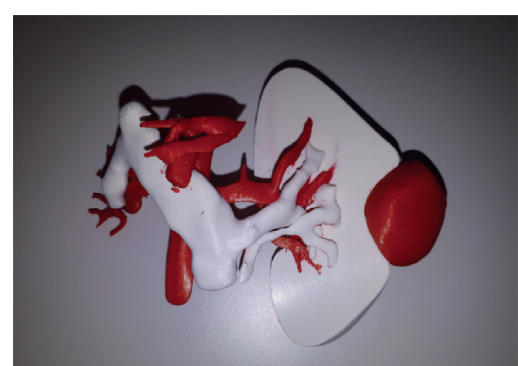

(c)

FIgURE 2: Three different visualization techniques for the MRI-based 3D models of patient 3. The patient has a transposition of the inferior vena cava below the superior mesenteric artery. (a) 2D screenshot of 3D rendering in 3D Slicer with a coronal T2-weighted MRI slice. (b) Augmented reality through the head-mounted display (HoloLens), holding the blue "cube" which allows for translation of the hologram. (c) 3D printed model printed with polylactic acid in Ultimaker S5, and the kidney is bisected.

understand how 3D modelling for preoperative planning influences pediatric surgical decision-making and confidence in order to understand how these models benefit our patients.

The proposed workflow appears useful during the preparation of NSS for bilateral or syndromic WT patients. In unilateral nonsyndromic patients, NSS is only performed in a very specific group of patients as controversies arise due to the inherent increased risk of positive surgical margins [3]. Current figures report a positive surgical margin of the sparingly removed tumor masses (treated with the Children's Oncology Group protocol) between 15.7\% and 31\% $[17,18]$. In accordance with the SIOP-RTSG Umbrella protocol, in the case of a positive surgical margin these young patients will need additional chemotherapy and possibly radiotherapy. In most cases, it is unknown how these positive margins occurred. The $3 \mathrm{D}$ visualization workflow may help surgeons to better understand complicated pathologic and anatomic regions and give an improved insight on where and how these positive margins occur. Additionally, 3D modelling may assist during the difficult patient selection for NSS through an increased understanding of the patient's anatomy. This twofold advantage might lead to fewer positive surgical margins and improved oncological outcomes.

In order to achieve improved clinical outcomes, all anatomical structures require a high-fidelity $3 \mathrm{D}$ model. However, the segmentation of the veins and UCS remains a manual and problematic task. The overlay of the arterial segmentation helps to differentiate between arterial and venous vasculature. Currently, this technique is insufficient for the accurate segmentation of intraparenchymal veins, likely due to intrasequential movement. The UCS was difficult to segment, as the full extent of the UCS is generally difficult to appreciate with standard imaging. More specific noncontrast imaging techniques such as noncontrast-enhanced MRVenography based on b-SSFP and MRUrography may help further improve the model quality and may speed up the segmentation. NC-MRV has the additional potential to allow for assessment of venous tumor thrombi [19]. Nevertheless, the arterial model is considered to have the highest surgical value as this is the most relevant for NSS
[4]. For this reason, despite the low model quality of the veins and UCS, the overall models were considered to be of sufficient value and usability.

In addition to accurate segmentations, the visualization of patient-specific 3D models is paramount to how the models are understood. Augmented reality visualization through a HoloLens offered a viable and fast technique for the visualization. A hologram is computed more easily and less costly in comparison to $3 \mathrm{D}$ printing. However, there is no consensus on whether there is a significant clinical advantage to the use of $A R$ instead of other visualization techniques such as $3 \mathrm{D}$ printing, $2 \mathrm{D}$ rendering on a computer monitor, or volume-rendering. Previously, no significant difference between $3 \mathrm{D}$ visualization techniques (AR or $3 \mathrm{D}$ printing) was found for WT patient-specific models [12]. However, we currently believe AR is the most desirable visualization technique due to the opportunity to develop Mixed Reality concepts. In the future, the HoloLens could allow intraoperative kidney-model matching. Mixed Reality models are used in adult laparoscopic renal surgery through registration of the 3D model with the laparoscopic image [7]. With Mixed Reality, rigid matching through an anatomical landmark registration has been described for open visceral surgery [20]. However, to the best of our knowledge, Mixed Reality for open renal surgery has not been described yet. In the context of NSS, this would allow the surgeon to get a sense of depth and infiltration of the tumor, and superimposing vasculature could assist surgeons to determine the resection margins.

Our pilot study is limited by the lack of evaluation of these anatomical models. A subjective analysis through questionnaires was not performed. However, we aimed to use novel imaging and visualization techniques and implement them into clinical care. The technique has been improved and should be further evaluated through the prospective use of the aforementioned questionnaires. Additionally, the 3D models should be compared with the pathology specimens in order to quantify the accuracy of the 3D models.

In the future, we aim to implement and evaluate 3D imaging technology in pediatric oncologic surgery as the standard of care and evaluate the added value. Additionally, 
we aim to develop more automated segmentation procedures for WT patients and work towards the use of intraoperative holograms through Mixed Reality.

\section{Conclusions}

This pilot study demonstrates how a strong collaboration between the pediatric surgery and radiology departments and 3D imaging specialists will help to shape the future of pediatric oncological surgery. A combination of specific high-resolution MRI sequences, protocolled segmentation techniques, and AR visualization improved the visualization for the preoperative planning of pediatric renal tumors. This designed 3D visualization workflow is an easily implementable technique to help pediatric oncological surgeons prepare for nephron-sparing surgery in patients with Wilms' tumors.

\section{Data Availability}

The .STL data used for the 3D models for this study are available from the corresponding author upon request.

\section{Conflicts of Interest}

The authors report no conflicts of interest.

\section{Acknowledgments}

The authors would like to acknowledge CARS 2020 for publishing this abstract in the Computer Assisted Radiology and Surgery Proceedings of the $34^{\text {th }}$ International Congress and Exhibition, Munich, Germany, June 23-27, 2020.

\section{References}

[1] N. Breslow, A. Olshan, J. B. Beckwith, and D. M. Green, "Epidemiology of Wilms tumor," Medical and Pediatric Oncology, vol. 21, no. 3, pp. 172-181, 1993.

[2] M. M. Van Den Heuvel-Eibrink, J. A. Hol, K. Pritchard-Jones et al., "Position paper: rationale for the treatment of Wilms tumour in the UMBRELLA SIOP-RTSG 2016 protocol," Nature Reviews Urology, vol. 14, no. 12, p. 743, 2017.

[3] J. C. H. Wilde, D. C. Aronson, B. Sznajder et al., "Nephron sparing surgery (NSS) for unilateral Wilms tumor (UWT): the SIOP 2001 experience," Pediatric Blood \& Cancer, vol. 61, no. 12, pp. 2175-2179, 2014.

[4] T. Klatte, V. Ficarra, C. Gratzke et al., "A literature review of renal surgical anatomy and surgical strategies for partial nephrectomy," European Urology, vol. 68, no. 6, pp. 980-992, 2015.

[5] C. Lupulescu and Z. Sun, "A systematic review of the clinical value and applications of three-dimensional printing in renal surgery," Journal of Clinical Medicine, vol. 8, no. 7, p. 990, 2019.

[6] N. Wake, M. A. Bjurlin, P. Rostami, H. Chandarana, and W. C. Huang, "Three-dimensional printing and augmented reality: enhanced precision for robotic assisted partial nephrectomy," Urology, vol. 116, pp. 227-228, 2018.

[7] E. Checcucci, D. Amparore, C. Fiori et al., "3D imaging applications for robotic urologic surgery: an ESUT YAUWP review," World Journal of Urology, vol. 38, no. 4, pp. 869-881, 2019.

[8] Ó. Girón-Vallejo, D. García-Calderón, R. Ruiz-Pruneda et al., "Three-dimensional printed model of bilateral Wilms tumor: a useful tool for planning nephron sparing surgery," Pediatric Blood Cancer, vol. 65, Article ID e26894, 2018.

[9] Á. Sánchez-Sánchez, Ó. Girón-Vallejo, R. Ruiz-Pruneda et al., "Three-dimensional printed model and virtual reconstruction: an extra tool for pediatric solid tumors surgery," $E$ uropean Journal of Pediatric Surgery Reports, vol. 6, no. 1, pp. e70-e76, 2018.

[10] J. Schenk, K.-L. Waag, N. Graf et al., "3D-visualization by MRI for surgical planning of Wilms tumors," RöFo-Fortschritte auf dem Gebiet der Röntgenstrahlen und der Bildgebenden Verfahren, vol. 176, no. 10, pp. 1447-1452, 2004.

[11] D. Zhang, G. Zeng, Y. Zhang et al., "3D reconstruction computed tomography scan in diagnosis of bilateral Wilm's tumor with its embolus in right atrium," Journal of X-Ray Science and Technology, vol. 24, no. 5, pp. 657-660, 2016.

[12] L. M. Wellens, J. Meulstee, C. P. van de Ven et al., "Comparison of 3-dimensional and augmented reality kidney models with conventional imaging data in the preoperative assessment of children with wilms tumors," JAMA Network Open, vol. 2, no. 4, Article ID e192633, 2019.

[13] N. Wake, T. Rude, S. K. Kang et al., "3D printed renal cancer models derived from MRI data: application in pre-surgical planning," Abdominal Radiology, vol. 42, no. 5, pp. 1501-1509, 2017.

[14] V. Vezhnevets and V. Konouchine, "GrowCut: interactive multi-label ND image segmentation by cellular automata," in Proceedings of the Graphicon, pp. 150-156, Novosibirsk, Russia, June 2005.

[15] N. Wake, J. S. Wysock, M. A. Bjurlin et al., "“Pin the tumor on the kidney:" an evaluation of how surgeons translate CT and MRI data to 3D models," Urology, vol. 131, pp. 255-261, 2019.

[16] D. D. Laganosky, C. P. Filson, and V. A. Master, "Surgical margins in nephron-sparing surgery for renal cell carcinoma," Current Urology Reports, vol. 18, p. 8, 2017.

[17] M. K. Richards, A. B. Goldin, P. F. Ehrlich et al., "Partial nephrectomy for nephroblastoma: a national cancer data base review," The American Surgeon, vol. 84, no. 3, pp. 338-343, 2018.

[18] A. M. Davidoff, R. B. Interiano, L. Wynn et al., "Overall survival and renal function of patients with synchronous bilateral Wilms tumor undergoing surgery at a single institution," Annals of Surgery, vol. 262, no. 4, pp. 570-576, 2015.

[19] L. Adams, B. Ralla, G. Engel et al., "Assessing venous thrombus in renal cell carcinoma: preliminary results for unenhanced 3D-SSFP MRI," Clinical Radiology, vol. 73, no. 8, pp. 757.e9-757.e19, 2018.

[20] R. Tang, L. Ma, C. Xiang et al., "Augmented reality navigation in open surgery for hilar cholangiocarcinoma resection with hemihepatectomy using video-based in situ three-dimensional anatomical modeling: a case report," Medicine (Baltimore), vol. 96, no. 37, Article ID e8083, 2017. 